\title{
Diffraction and depths-of-field effects in electron beam imaging at SURF III
}

\author{
U. Arp* \\ Synchrotron Ultraviolet Radiation Facility (SURF), National Institute of Standards and Technology (NIST), \\ 100 Bureau Drive, MS 8410, Gaithersburg, MD 20899-8410, USA
}

Received 26 June 2000; received in revised form 2 October 2000; accepted 10 October 2000

\begin{abstract}
Imaging an electron beam with visible light is a common method of diagnostics applied to electron accelerators. It is a straightforward way to deduce the transverse electron distribution as well as its changes over time. The electrons stored in the Synchrotron Ultraviolet Radiation Facility (SURF) III at the National Institute of Standards and Technology (NIST) were studied over an extended period of time to characterize the upgraded accelerator. There is good agreement between experimental and theoretical horizontal beam sizes at three different electron energies. (C) 2001 Elsevier Science B.V. All rights reserved.
\end{abstract}

PACS: 42.25.Fx; 42.30.-d; 41.75.Fr; 41.85.Ew

Keywords: Electron beam diagnostics; Transverse beam size; Diffraction; Depth of field; Synchrotron radiation; Storage ring

\section{Introduction}

After the upgrade of the Synchrotron Ultraviolet Radiation Facility at NIST to SURF III [1], it became necessary to study the accelerator performance in different operating conditions. This upgrade included the total replacement of the magnet system, refurbishment of the magnet power supply, improvements of the SURF vacuum chamber, a new RF transmitter, and the implementation of a software-based control system. The newly configured storage ring can operate at energies up to $361 \mathrm{MeV}$, limited

\footnotetext{
*Fax: + 1-301-208-6937.

E-mail address: uwe.arp@nist.gov (U. Arp).
}

currently by the available RF power. One of the most important parameters is the transverse size of the electron beam, which is directly related to basic accelerator parameters. A good example on how to measure transverse beam size was given by Andersson and Tagger [2], who in a very detailed analysis explained the mechanism of using visible light to determine the beam size at MAX in Lund. Several other authors gave more general descriptions of diffraction and depth-of-field contributions in measuring electron beam sizes [3-6]. It has further been shown that the two effects can be treated consistently, as a single phenomenon, within the frame of classical electrodynamics applied to the emission and focussing of SR [7,8]. However, in this paper we will follow the simple model given in Ref. [2]. 
The theoretical beam size can easily be calculated for a weak focusing machine like SURF and compared to the results from the measurements. The three parameters describing the SURF lattice completely are the magnetic field index, the orbit radius and the electron energy. The field index has been measured through magnetic field mapping [1] to be $n=\left.\left(-\rho_{0} / B_{0}\right)(\partial B / \partial r)\right|_{r=\rho_{0}}=0.594 \pm 0.006$. In a circular single-magnet machine, the radius of the electron orbit $\rho_{0}$ is determined by the RF frequency $v_{\mathrm{RF}}$,

$\rho_{0}=\frac{\beta h c}{2 \pi v_{\mathrm{RF}}}$

where $h=2$ is the harmonic number or number of electron bunches. $\beta c$ is the relativistic speed of the electrons. The RF frequency at SURF III is $v_{\mathrm{RF}}=113.847 \mathrm{MHz}$ leading to a radius of the electron orbit $\rho_{0}=838.2 \mathrm{~mm}$. The electron energy can be deduced from magnetic flux density measurements

$E_{0}=B_{0} \beta c \rho_{0} e=\frac{B_{0}(\beta c)^{2} e h}{2 \pi v_{\mathrm{RF}}}$

using the already known radius or RF frequency.

The vertical angular distribution of magnetobremsstrahlung or synchrotron radiation is important for the experimental and theoretical analysis, because it plays a central role both for depth-of-field and diffraction effects in beam size measurements. Wiedemann $[9,10]$ gives a good review on the origins and properties of synchrotron radiation in his books on accelerator physics. To a good approximation the vertical opening half-angle $\psi_{\text {typ }}$ depends only on the bending radius and the wavelength $\psi_{\text {typ }}=\sqrt[3]{3 \lambda / 4 \pi \rho_{0}}$, if $\lambda \gg \lambda_{\mathrm{c}}$ (the critical wavelength). For SURF III at $\lambda=550 \mathrm{~nm}$ this value is $0.309^{\circ}$.

\section{Theory}

For a weak focusing storage ring like SURF III the horizontal electron beam size can be calculated using a few basic parameters. The horizontal beam size can be calculated using the following well-known equation [9]

$\sigma_{\mathrm{x}}=\sqrt{\varepsilon_{\mathrm{x}} \beta_{\mathrm{x}}+\left(\frac{\sigma_{\mathrm{E}}}{E_{0}}\right)^{2} \eta_{\mathrm{x}}^{2}}$.

$\varepsilon_{\mathrm{x}}=C_{\mathrm{q}} \gamma^{2}(1 / n \sqrt{1-n})$ is the horizontal emittance,

$\beta_{\mathrm{x}}=\rho_{0} / \sqrt{1-n}$ the horizontal beta function, $\sigma_{\mathrm{E}} /$ $E_{0}=\sqrt{C_{\mathrm{q}} \gamma^{2} \rho_{0}^{-1}(1-n / 3-4 n)}$ the energy spread and $\eta_{\mathrm{x}}=\rho_{0} / 1-n$ the horizontal dispersion function. Substituting these expressions into Eq. (3) leads to a simple expression for the horizontal beam size at SURF:

$$
\begin{aligned}
\sigma_{\mathrm{x}} & =\gamma \sqrt{\frac{-3 C_{\mathrm{q}} \rho_{0}(n-1)^{2}}{4 n^{4}-11 n^{3}+10 n^{2}-3 n}} \\
& =\gamma \sqrt{\frac{3 C_{\mathrm{q}} \rho_{0}}{3 n-4 n^{2}}} \approx 1.612 \mu \mathrm{m} \gamma
\end{aligned}
$$

with the quantum excitation constant [11] $C_{\mathrm{q}}=55 h_{\mathrm{p}} / 64 \sqrt{3} \pi m_{\mathrm{e}} c=3.832 \times 10^{-13} \mathrm{~m}$. The horizontal beam size grows proportional to the energy, effectively limiting the energies accessible with a weak focusing accelerator because of the horizontal aperture determined by the vacuum chamber and RF cavity. The theoretical vertical beam size without coupling of the horizontal and vertical betatron motions is given by

$\sigma_{\mathrm{y}}=\sqrt{\varepsilon_{\mathrm{y}} \beta_{\mathrm{y}}}=\sqrt{\frac{C_{\mathrm{q}} \rho_{0}}{n}}=0.735 \mu \mathrm{m}$

independent of all operating parameters but the orbit radius and the field index. In reality, the vertical beam size is bigger, because of coupling effects and emittance growth due to rest gas scattering, where coupling is the dominant effect.

\section{Experiment}

The beam size measurements were performed at beamline 6 at SURF III. A spherical symmetric lens was used, as shown in Fig. 1. If used with a narrow-band interference filter, a perfect spherical lens images almost without distortions if the object and image distance are the same. We used an interference filter with center wavelength $\lambda_{0}=550 \mathrm{~nm}$ and transmission FWHM of $\Delta \lambda=10 \mathrm{~nm}$. Within the bandwidth of this filter, 


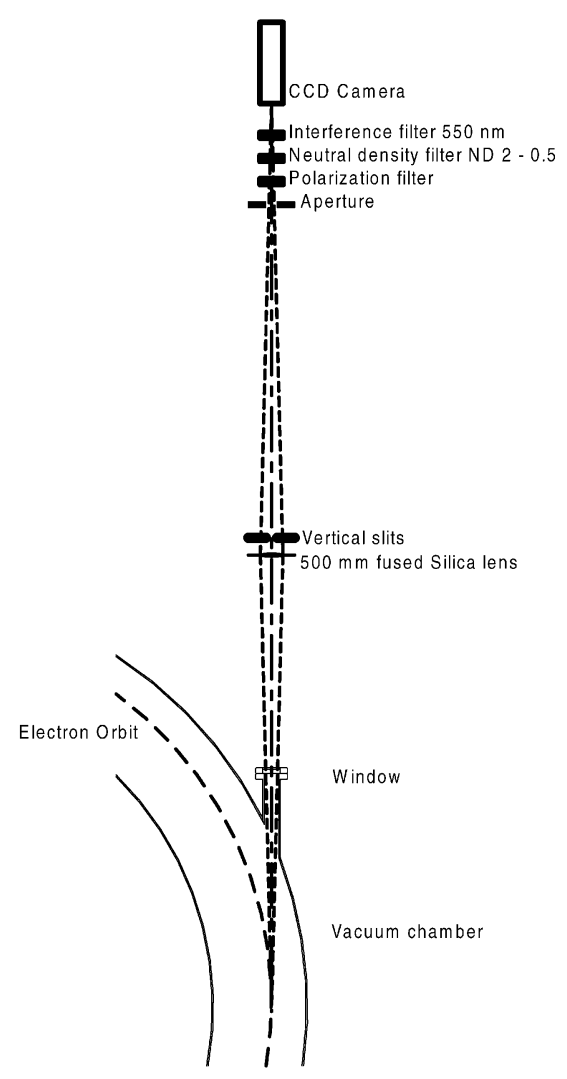

Fig. 1. Top view of the imaging setup at beamline 6 at SURF III.

the transmission was $50 \%$. In addition, we employed various neutral density filters to control the incident intensity and polarization filters to select different polarization directions. Two different CCD cameras were used: A cooled scientific CCD with 12-bit resolution and later a simpler analog camera in conjunction with an eight-bit frame grabber. Following the simplified model given in Ref. [2], two major contributions have to be considered in the analysis of the imaging results: depth-of-field and diffraction effects.

\subsection{Depth of field}

Fig. 2 illustrates the coordinate system for the imaging setup used in our experiment. A spherical symmetric lens is positioned at a distance $o$ from the source and an imaging detector is placed at the distance $i$ from the lens (we used $o=i=1000 \mathrm{~mm}$; conjugate ratio of 1). The horizontal aperture of the lens determines the source depth or length. The distance from the tangent point $o$ and the horizontal half-slit widths $x_{\mathrm{h}}$ determine the horizontal acceptance angle $\xi=2 \arctan \left(x_{\mathrm{h}} / o\right)$. Fig. 3 shows the relationship between the horizontal acceptance angle $\xi$, the arc length $l$, chord $a$, and height $h$.

$l=\rho_{0} \xi, \quad a=2 \rho_{0} \sin \left(\frac{\xi}{2}\right), h=\rho_{0}\left(1-\cos \left(\frac{\xi}{2}\right)\right)$.

Table 1 lists the properties of the imaging system for different horizontal slit sizes (the slit position is at the lens). When we use a fairly large horizontal aperture, the beam imaging is out of focus over a wide range. The imaging only works perfectly for the source position $\mathrm{S}$ (see Figs. 2 and 3). For the range from $-a / 2$ to $a / 2$, the vertical angular divergence of the beam will distort the image. If we assume a contribution from the beam size and the vertical angular divergence of the emitted photons over the source depths, a simple approximation can be formulated for the vertical image cross-section

$$
\begin{aligned}
I_{\mathrm{dof}}\left(y^{\prime}\right)= & \int_{-a / 2}^{a / 2} \frac{1}{\sqrt{2 \pi}} \frac{o}{\mathrm{i} \sqrt{\sigma_{\mathrm{y}}^{2}+z^{2} \sigma_{\psi}^{2}}} \\
& \times \exp \left[\frac{-y^{\prime 2}}{2} \frac{o^{2}}{\mathrm{i}^{2}\left(\sigma_{\mathrm{y}}^{2}+z^{2} \sigma_{\psi}^{2}\right)}\right] \mathrm{d} z
\end{aligned}
$$

in which $\sigma_{\mathrm{y}}$ is vertical beam size (Gaussian value and to be precise the diffraction width of the image) and $\sigma_{\psi}$ is the Gaussian width of the vertical angular spread. $\sigma_{\psi}$ was deduced from a fit to calculated vertical profiles employing two Gaussians. The solid line in Fig. 4 is the calculated vertical angular spread for SURF III at $\lambda=550 \mathrm{~nm}$, for the sum of parallel and perpendicular polarization. The longdashed line is the result of a fit consisting of two Gaussian profiles of equal width and the shortdashed lines are the individual Gaussians. The width parameter deduced from the fitting procedure was $\sigma_{\psi}^{\prime}=0.14^{\circ}$ for each of the profiles. There is no closed analytical form for Eq. (7), so the results were found numerically.

The solid line in Fig. 5 is a measured vertical profile (both parallel and perpendicular polarization), which was collected at $330.7 \mathrm{MeV}$ without a 


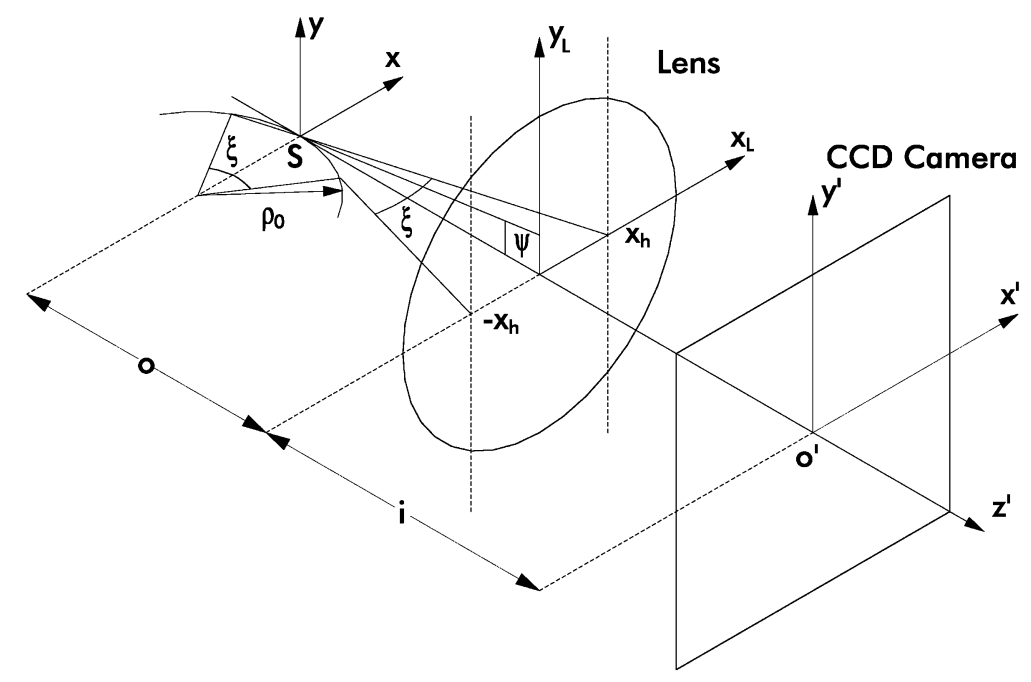

Fig. 2. Definition for the data analysis in the beam imaging experiment. S, source point; $\rho_{0}$, orbital radius; $o$, distance object lens; $i$, distance lens camera; $\xi$, horizontal acceptance angle given by the horizontal lens half-aperture $x_{\mathrm{h}} ; \psi$, natural vertical opening angle of the synchrotron radiation.

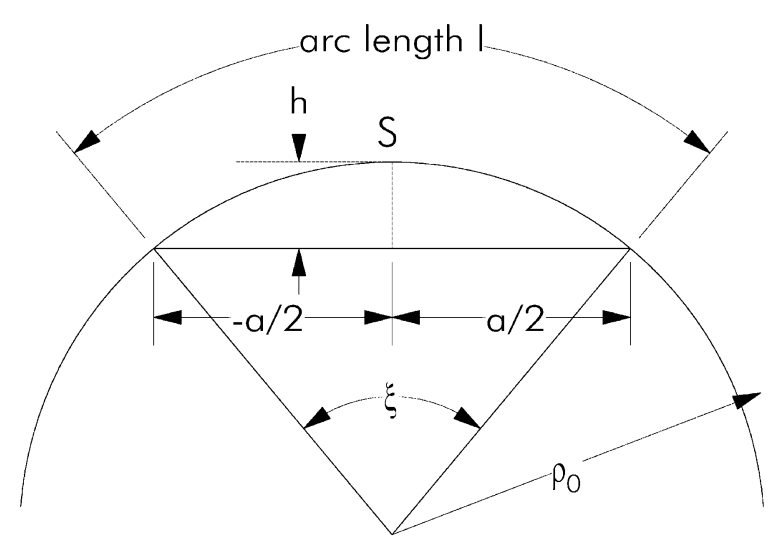

Fig. 3. Relationship between the horizontal acceptance angle $\xi$, the arc length $l$, chord $a$, and height $h$.

slit (horizontal opening $x_{\mathrm{h}}=25.4 \mathrm{~mm}$ ). The influence of the depth-of-field effect is apparent and the measured profile departs far from a Gaussian. The dashed line is the result of a calculation using Eq. (7) with $\sigma_{\psi}=2 \sigma_{\psi}^{\prime}$ and $\sigma_{\mathrm{y}}=21 \mu \mathrm{m}$. These parameters were adjusted to give the best result to the eye. The dashed-tripledotted curve is the Gaussian with $\sigma_{\mathrm{y}}=21 \mu \mathrm{m}$ to illustrate the huge difference caused by depth-offield effects and the dashed-dotted line is the profile measured with $x_{\mathrm{h}}=3.175 \mathrm{~mm}$.

\subsection{Diffraction effects}

Diffraction limits the vertical image resolution considerably. Equations for diffraction by a rectangular slit can be found for example in Menzel's collection of formulas [12]. In Fig. 6, the vertical diffraction of a point source is illustrated (solid line) and a Gaussian (dashed line) fit to the calculated profile to deduce the width. The aperture width on the lens is given horizontally by the slits width $x_{\mathrm{h}}$ and vertically by the natural emission angle of the synchrotron radiation. The typical vertical emission angle is

$\psi_{\text {typ }}=\sqrt[3]{\frac{3 \lambda}{4 \pi \rho_{0}}}=0.309^{\circ}$

(illustrated by the vertical lines in Fig. 4). The equation for the diffraction of a point source by a rectangular slit is

$I=\left(\frac{\sin (\alpha)}{\alpha}\right)^{2}\left(\frac{\sin (\beta)}{\beta}\right)^{2}$

with $\alpha=(\pi / \lambda) a \sin (\xi)$ and $\beta=(\pi / \lambda) b \sin (\psi)$ $=\sqrt[3]{6 \pi^{2} o^{3} / \lambda^{2} \rho_{0} \sin (\psi)}$, where $a$ is the horizontal slit width, given by $a=2 x_{\mathrm{h}}$, and $b$ the vertical slit width, using Eq. (8) given by $b \approx 2 o \psi_{\text {typ. Using }}$ this simple estimation of the diffraction effect one 
Table 1

Properties of the imaging system used at SURF's beamline 6. The radius of the electron orbit is $\rho_{0}=838.2 \mathrm{~mm}$. The object and image distance are $1000 \mathrm{~mm}$

\begin{tabular}{llccc}
\hline $\begin{array}{l}\text { Horizontal half-slit } \\
\text { widths } x_{\mathrm{h}}(\mathrm{mm})\end{array}$ & $\begin{array}{l}\text { Horizontal acceptance } \\
\text { angle } \xi\left({ }^{\circ}\right)\end{array}$ & Arc length/(mm) & Chord $a(\mathrm{~mm})$ & Height $h(\mathrm{~mm})$ \\
\hline 25.4 & 2.91 & 42.571 & 42.567 & 0.27 \\
12.7 & 1.455 & 21.289 & 21.289 & 0.068 \\
6.35 & 0.728 & 10.645 & 10.645 & 0.017 \\
3.175 & 0.364 & 5.323 & 5.323 & 0.004 \\
1.5875 & 0.182 & 2.661 & 2.661 & 0.001 \\
\hline
\end{tabular}

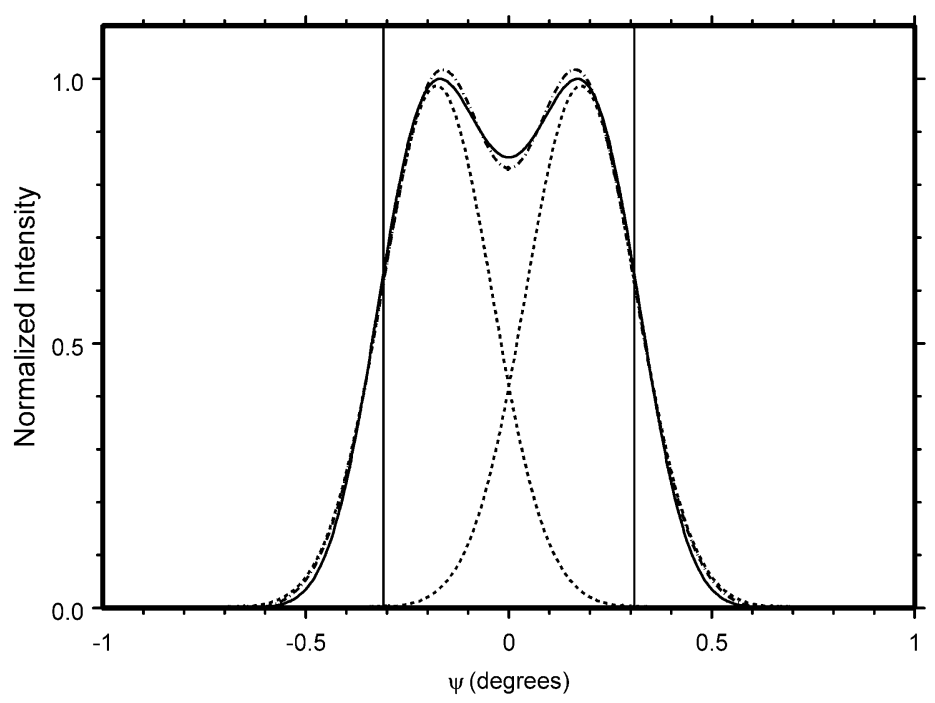

Fig. 4. The solid line is the calculated vertical angular spread for SURF III at $\lambda=550 \mathrm{~nm}$. The long-dashed spectrum is the result of a fit consisting of two Gaussian profiles of equal width and the short-dashed lines are the individual Gaussians. The width parameter deduced from the fitting procedure was $\sigma_{\psi}^{\prime}=0.14^{\circ}$ for each of the profiles.

can see that, without depth-of-field effects, the image of a point source would appear almost as a Gaussian profile with $\sigma_{\mathrm{D}} \approx 18 \mu \mathrm{m}$ (a more detailed analysis can be found in Ref. [5]). The real source image of an extended source is the convolution of the Gaussian source profile with the diffraction contribution. The convolution of two Gaussian profiles with widths $\sigma_{1}$ and $\sigma_{2}$ results in a Gaussian with $\sigma_{\text {tot }}=\sqrt{\sigma_{1}^{2}+\sigma_{2}^{2}}$. Together with the depth-of-field analysis, we now developed the means to analyze our images.

\section{Results}

To determine the validity of the analysis, experiments were performed with wide vertical slits $\left(x_{\mathrm{h}}=25.4 \mathrm{~mm}\right)$ and narrow slits $\left(x_{\mathrm{h}}=3.175 \mathrm{~mm}\right)$.

\subsection{Vertical beam size}

The analysis done before allows us to deduce the vertical beam size from the distorted data. The curves in Fig. 5 were used to determine the vertical 


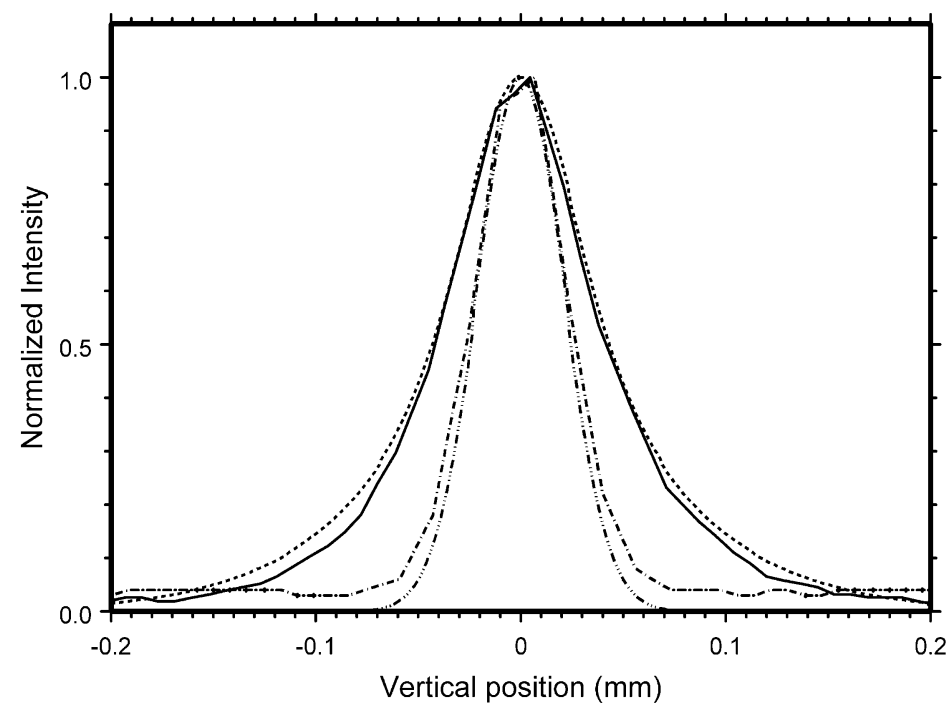

Fig. 5. The solid line is a measured vertical profile (both parallel and perpendicular polarization), which was collected at $330.7 \mathrm{MeV}$ without a slit (horizontal opening $x_{\mathrm{h}}=25.4 \mathrm{~mm}$ ). The influence of the depth-of-field effect is apparent and the measured profile departs far from a Gaussian. The dashed line is the result of a calculation using Eq. (7) with $\sigma_{\psi}=2 \sigma_{\psi}^{\prime}$ and $\sigma_{\mathrm{y}}=21 \mu \mathrm{m}$. These parameters were adjusted to give the best result to the eye. The dashed-triple-dotted curve is the Gaussian with $\sigma_{\mathrm{y}}=21 \mu \mathrm{m}$ to illustrate the huge difference caused by depth-of-field effects and the dashed-dotted line is the profile measured with $x_{\mathrm{h}}=3.175 \mathrm{~mm}$.

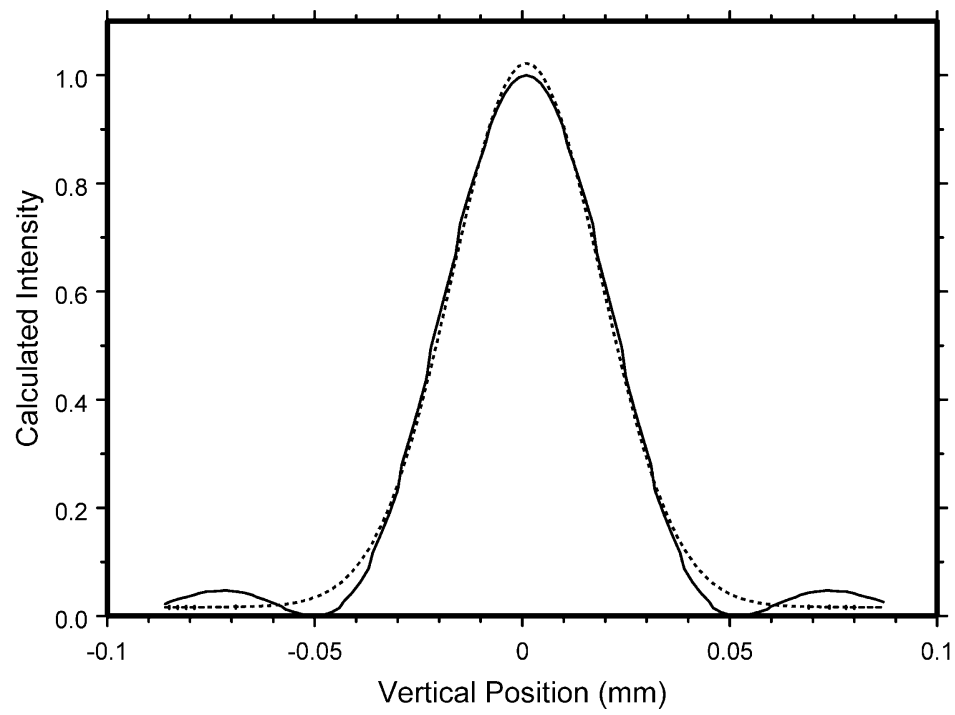

Fig. 6. The solid line the calculated vertical diffraction of a point and the dashed line is a Gaussian fit to the calculated profile to deduce the width.

beam size. The Gaussian width parameter deduced from a fit to the experimental profile is $\sigma_{\mathrm{y}}=22.5 \mu \mathrm{m}$. If the diffraction width $\sigma_{\mathrm{D}}=18 \mu \mathrm{m}$ is considered, the real vertical beam cross-section becomes $\sigma_{\mathrm{y}}=13.5 \mu \mathrm{m}$. The full-width at half- maximum of the beam is $32 \pm 4 \mu \mathrm{m}$ at 330.7 MeV. This vertical size is considerably larger than the calculated value in Eq. (7) because of the coupling between the horizontal and vertical betatron motions. 


\subsection{Horizontal beam size}

Diffraction effects are negligible for horizontal beam-size measurements, because of their small magnitude compared to the horizontal size. And if the small vertical slits are used, the depth-of-field contribution is negligible as well and we can easily determine the beam size. The two results from fits to the data with $x_{\mathrm{h}}=25.4$ and $3.175 \mathrm{~mm}$ are $\sigma_{\mathrm{H}}=$ 1.048 and $1.036 \mathrm{~mm}$, respectively. The second value is more accurate and leads to FWHM of $2.439 \mathrm{~mm}$. With that the aspect ratio of the beam is about $76: 1$ at $330.7 \mathrm{MeV}$. In Table 2, measured and calculated horizontal beam sizes are compared for three different energies. The agreement between theory and experiment is good.

\subsection{Fuzz conditions}

At low electron energies, the electron beam lifetime is dominated by Touschek scattering [9]. The Touschek lifetime is proportional to the electron bunch volume. For this reason, the vertical betatron oscillation is excited in SURF to extend the vertical dimension of the beam. At

\section{Table 2}

Calculated and experimental horizontal beam size FWHM at different electron energies. The uncertainties are standard deviations deduced from the fitting procedure

\begin{tabular}{lll}
\hline $\begin{array}{l}\text { Electron energy } \\
(\mathrm{MeV})\end{array}$ & $\begin{array}{l}\text { Experimental FWHM } \\
(\mathrm{mm})\end{array}$ & $\begin{array}{l}\text { Calculated FWHM } \\
(\mathrm{mm})\end{array}$ \\
\hline 183.1 & $1.367 \pm 0.003$ & $1.37 \pm 0.008$ \\
284.4 & $2.087 \pm 0.003$ & $2.11 \pm 0.013$ \\
330.7 & $2.471 \pm 0.004$ & $2.45 \pm 0.015$ \\
\hline
\end{tabular}

SURF, this is called applying fuzz [13] to the beam because it induces random vertical motion of the electrons. Beam-size results for three different fuzz conditions at $284.4 \mathrm{MeV}$ electron energy are listed in Table 3. In this case, we used a polarization filter to either select the strong horizontal (parallel to the electron orbit) or weaker vertical (perpendicular to the electron orbit) polarization components of the synchrotron radiation emitted at $550 \mathrm{~nm}$. The resolving power of the optical system is best for the horizontal polarization, because of the smaller vertical angular spread [2]. In Fig. 7, the horizontal (top) and vertical (bottom) polarization components are shown for a fully fuzzed beam (11.5 W fuzz power at $284.4 \mathrm{MeV})$.

\section{Conclusions}

The transverse electron beam size was determined for SURF III. The vertical full-width at half-maximum was determined to be $32 \pm 4 \mu \mathrm{m}$ at $330.7 \mathrm{MeV}$ electron energy. This value is completely determined by the coupling between the horizontal and vertical betatron oscillations. A simple theoretical model to include depth-of-field and diffraction effects was successfully developed to deduce the vertical beam size. The horizontal beam size was determined for three electron energies and found to agree well with calculated values.

\section{Acknowledgement}

Support by the staff of the SURF III facility is gratefully acknowledged.

Table 3

Experimental vertical and horizontal beam sizes depending on the fuzz power at $284.4 \mathrm{MeV}$ electron energy, both measured using only the horizontal polarization component and the total unpolarized radiation. All values are full-width at half-maximum and not corrected for diffraction. The excitation of the vertical betatron oscillation mixes the perpendicular and parallel polarization components to some extend. Also not only random motion of the electrons is induced, but also coherent motion further distorting the imaging results. The uncertainties are standard deviations deduced from the fitting procedure

\begin{tabular}{llllll}
\hline Fuzz power $(\mathrm{W})$ & \multicolumn{3}{l}{ Horizontal polarization } & & \multicolumn{2}{l}{ Total polarization } \\
\cline { 2 - 3 } & FWHM $_{\text {HOR }}(\mathrm{mm})$ & FWHM $_{\text {VER }}(\mathrm{mm})$ & & FWHM $_{\text {HOR }}(\mathrm{mm})$ & FWHM \\
\hline 0 & $1.491 \pm 0.002$ & $0.045 \pm 0.002$ & $2.023 \pm 0.005$ & $0.052 \pm 0.002$ \\
4 & $3.101 \pm 0.012$ & $1.528 \pm 0.002$ & $3.530 \pm 0.019$ & $2.355 \pm 0.007$ \\
11.5 & $2.880 \pm 0.007$ & $2.531 \pm 0.017$ & & $3.160 \pm 0.009$ & $3.193 \pm 0.045$ \\
\hline
\end{tabular}



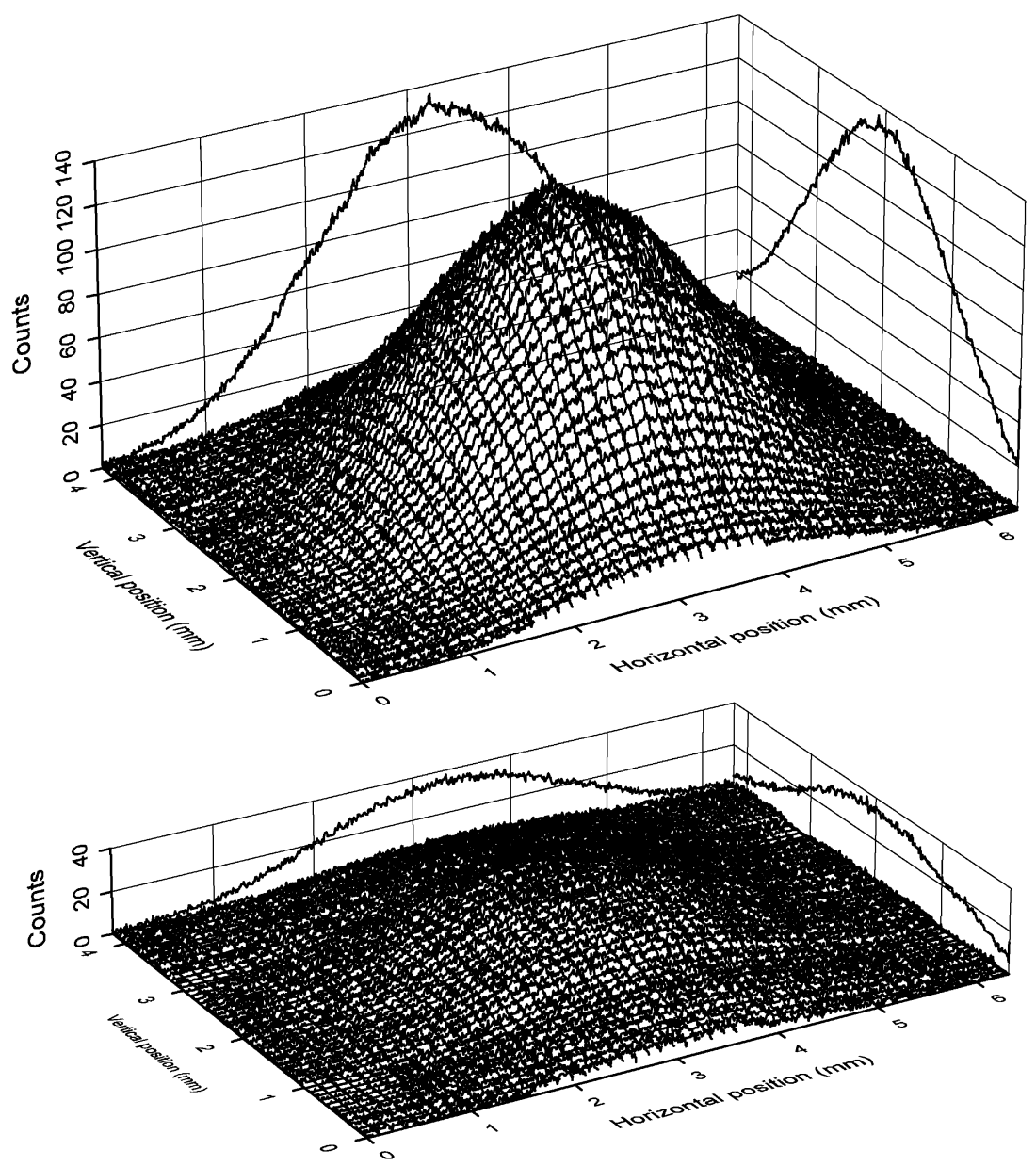

Fig. 7. Top: Surface plot of the horizontal polarization component at $284.4 \mathrm{MeV}$ with full fuzz. Bottom: Plot of the vertical polarization component.

\section{References}

[1] M.L. Furst et al., in: A. Luccio, W. MacKay (Eds.), Proceedings of the 1999 Particle Accelerator Conference, New York, IEEE, New York, 1999.

[2] А․ Andersson, J. Tagger, Nucl. Instr. and Meth. A 364 (1995) 4.

[3] A. Hofmann, Beam Diagnostics and Applications, in; R.O. Hefferl, S.R. Smith, J.D. Masek (Eds.), Beam Instrumentation Workshop, AIP Conf. Proc., AIP, Woodbury, New York, 1998.

[4] A. Hofmann, F. Méot, Nucl. Instr. and Meth. A 203 (1982) 483.

[5] A. Ogata, Nucl. Instr. and Meth. A 301 (1991) 596.

[6] W.B. Peatman, K. Holldack, J. Synchrotron Rad. 5 (1998) 639.
[7] O. Chubar, Proceedings of the 1993 IEEE Particle Accelerator Conference, Washington, DC, 1994.

[8] Å. Andersson, Electron beam profile measurements and emittance manipulation at the MAX-laboratory, Ph.D. thesis, Lund University, 1997.

[9] H. Wiedemann, Particle Accelerator Physics, Springer, New York, 1993.

[10] H. Wiedemann, Particle Accelerator Physics II, Springer, New York, 1995.

[11] M. Sands, in: B. Touschek (Ed.), Physics With Intersecting Storage Rings, Academic Press, New York, 1971.

[12] D.H. Menzel, Fundamental Formulas of Physics, Dover, New York, 1960.

[13] G. Rakowsky, IEEE Trans. Nucl. Sci. NS-26 (1979) 3845. 